\title{
Viscosupplementation in the hip: evaluation of hyaluronic acid formulations
}

\author{
M. P. J. van den Bekerom $\cdot$ B. Rys $\cdot$ M. Mulier
}

Received: 17 November 2006 / Published online: 16 June 2007

(C) Springer-Verlag 2007

\begin{abstract}
This study compares three different hyaluronate formulations and evaluates functionality, time of satisfactory pain relief and also the delay in performing a total hip arthroplasty. One hundred and twenty patients (126 hips) received viscosupplementation with one of the three hyaluronate formulations. All patients were candidate for surgical treatment with a total hip arthroplasty. Three different products were consecutively used: Adant ${ }^{\circledR}$, Synocrom ${ }^{\circledR}$ or Synvisc ${ }^{\circledR}$. Patients were assessed 6 weeks after each infiltration using Visual Analogue Scale and Harris Hip Score. The Harris Hip Score increased significantly in two of the three groups compared to baseline, but no statistical significant difference was noted between the groups. Viscosupplementation provides significant pain reduction in two of the three groups. There is no significant difference in duration of the effect of the first infiltration between the three groups. The positive effect was still ongoing at the end point of the study in 46 hips: $51 \%$ of the patients did not undergo total hip arthroplasty, 3 years after viscosupplementation.
\end{abstract}

M. P. J. van den Bekerom

Department of Orthopaedic and Trauma Surgery,

Gelre Hospitals, Apeldoorn, The Netherlands

B. Rys $\cdot$ M. Mulier

Department of Orthopaedic Surgery,

UZ Pellenberg, Lubbeek, Belgium

M. Mulier ( $\square)$

Department of Orthopaedic Surgery, UZ Pellenberg,

K.U.Leuven, Weligerveld 1, 3212 Pellenberg, Belgium

e-mail: michiel.mulier@uz.kuleuven.ac.be
Keywords Hyaluronic acid · Hyaluronan - Injections · Intra-articular · Viscosupplementation · Hip osteoarthritis . Non-operative treatment

$\begin{array}{ll}\text { Abbreviations } \\ \text { OA } & \text { Osteo-arthritis } \\ \text { NSAID } & \text { Non steroidal anti-inflammatory drug } \\ \text { VS } & \text { Viscosupplementation } \\ \text { HA } & \text { Hyaluronic acid } \\ \text { THA } & \text { Total hip arthroplasty } \\ \text { HHS } & \text { Harris hip score } \\ \text { VAS } & \text { Visual analogue scale } \\ \text { MW } & \text { Molecular weight }\end{array}$

\section{Introduction}

Severe osteoarthritis (OA) of the hip is characterized by pain and reduced mobility. It affects primarily adults aged over 50 years, but the age of onset of hip OA linked complaints seems to decrease over the last decennia [12]. First-line treatment consists of pain reduction with analgesics and non steroidal anti inflammatory drugs (NSAID). Rehabilitation treatment, education and the use of walking aids can help providing pain relief and improving mobility. When those therapeutic options fail or the analgesic treatment causes intolerable side effects, joint lavage, hip osteotomy or total hip arthroplasty (THA) can be considered. This arthroplasty technique has greatly improved and patients experience pain reduction and improvement of the quality of life. However, the intervention still requires prolonged anaesthesia, and is followed by a long-term rehabilitation program. Though complications are rare and protheses have an increasing duration of life, reinterventions are still needed [4]. 
Viscosupplementation (VS) is the administration of hyaluronan and/or hyaluronic acid preparations to joint synovial fluid for the treatment of OA in order to restore the biologic properties of normal hyaluronic acid (HA).

The use of VS with HA was first described to provide pain relief and to increase mobility of the knee joint. The VS is an effective treatment for OA of the knee with beneficial effects on pain, function and patient global assessment [14]. HA products have more prolonged effects than intraarticular corticosteroids [14]. Since 1984, this technique is also used for the management of OA of the hip joint [2]. In the available literature there is no consensus on the number of injections, the dosage per injection, the most appropriate formulation of HA, and the optimal method for controlling the needle positioning in the hip joint. Moreover, the patient selection criteria differ from one study to another. To our knowledge there is no documentation available on the possible time gain for patients treated with VS of the hip joint before they have to undergo THA.

The results of a trial comparing three formulations of HA were presented. All patients were candidates for THA. The time of satisfactory pain relief, improved functionality obtained with each infiltration of HA and the delay in surgery were evaluated.

\section{Patients and methods}

Patient selection

The use of VS in patients with severe OA is common practise in our hospital, thus not requiring approval of the ethical committee. Patients gave their informed consent for this prospective study. Between March 2001 and February 2005, 120 patients (126 hips), 49 males and 71 females, responding to the eligibility criteria listed below, received viscosupplementation. Three different products were consecutively used: Adant $^{\circledR}$ (Tedec-Meiji Failma, Madrid, Spain) (Group 1) (Synthetic hyaluronic acid with an average molecular weight of 0.6-1.2 million Da), Synocrom ${ }^{\circledR}$ (Croma Pharma, Leobendorf, Austria) (Group 2) (Sodium hyaluronate with a average molecular weight of 1.6 million Da) or Synvisc ${ }^{\circledR}$ (Altana Pharma, Konstanz, Germany) (Group 3) (Hylan GF 20 with an average molecular weight of 6.0 million Da) (Table 1).

\section{Eligibility criteria}

- Age between 30 and 70 years and suffering idiopathic radiologically confirmed hip OA.

- Visual Analogue Scale (VAS) score for pain greater then 30 (on a 100-point scale; 0 no pain and 100 "the worst pain imaginable")

- Have persistent pain for longer than 1 month despite use of analgesics or NSAID's.

- Be candidate for surgical treatment with a THA, according to the following criteria:

- continuous hip pain, also during the night, requiring daily intake of NSAID's or pain medication

- disabled gait pattern and need of walking aid

- Be able to understand the information relative to viscosupplementation and to give informed consent.

\section{Exclusion criteria}

- Pregnancy

- Contraindications to intra-articular hyaluronic-acid preparations

- Major hip dysplasia or congenital abnormality of the hip

- Patients with systemic corticosteroids or intra-articular corticosteroid injections in the last 6 months

- Contra-lateral THA or hip arthroscopy in the last 6 months

- Oral or parenteral anticoagulant therapy

- Previous hyaluronic acid hip infiltrations

- Skin diseases or infections

- Signs of haemarthrosis

- History of allergy or hypersensitivity to iodated contrast

Treatment

Patients received an intra articular infiltration with one of the three products. The manufacturer's treatment recommendations were followed. Patients having initially experienced a satisfactory pain relief are offered a second and third infiltration or THA when the condition deteriorates. Injection of the viscosupplementation was performed under sterile conditions by the same experienced orthopaedic surgeon $(\mathrm{MM})$ in all patients. After skin cleaning a lumbar puncture needle was inserted in a lateral approach. Layer by
Table 1 Characteristics of infiltrated population

\begin{tabular}{|c|c|c|c|c|}
\hline & Period of treatment & Patients & Gender & Age \\
\hline Adant & $27-11-02$ till 23-02-05 & 91 & 35 s and 65 q & $61.8 \pm 12.8$ \\
\hline Synocrom & 19-01-04 till 04-10-04 & 20 & 7 ô and 13 q & $62.1 \pm 14.5$ \\
\hline Synvisc & $15-03-01$ till $24-04-03$ & 15 & $7 \hat{o}^{-1}$ and 8 + & $61.9 \pm 15.3$ \\
\hline
\end{tabular}


layer local anaesthesia was performed using lidocaine $1 \%$. Iodinated contrast agent Ultravist ${ }^{\circledR}$ (Schering, Berlin, Germany) was injected. The needle positioning into the joint cavity was fluoroscopically controlled (Fig. 1). Arthrocentesis was carefully performed prior to each injection to remove any effusion.

After resting for $2 \mathrm{~h}$, the patient was allowed to walk and to return home. The patient was advised to rest at home until the next morning.

Oral symptomatic slow acting drugs for osteoarthritis were authorized if they were taken at a stable dose for more than 3 months prior to inclusion in the study. These analgesics were continued at a stable dose during the VS treatment.

\section{Evaluation}

All patients were assessed at baseline and 6 weeks after each infiltration. During this consultation in the outpatient clinic, the pain and functionality were evaluated using the VAS pain during walking score (100-point scale) and the Harris Hip Score (HHS). The latter is a clinical scoring system on a total of 100 points whereby the following subscales are rated: function (47 points), pain (44 points),
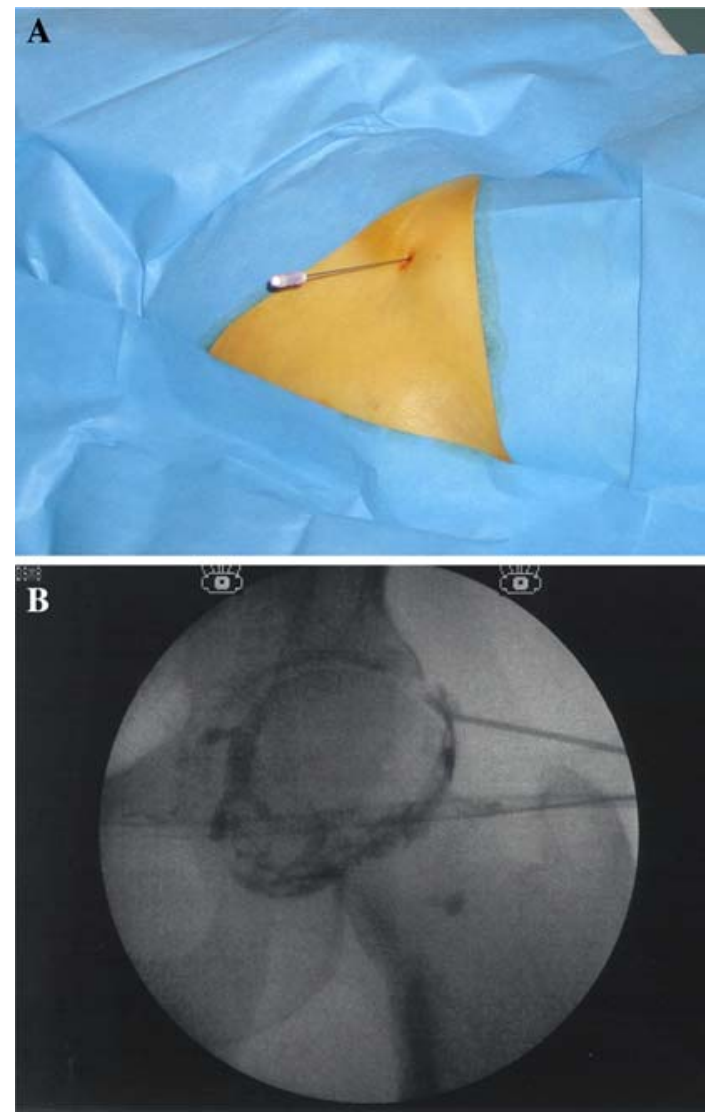

Fig. 1 a Needle placement for injection of the viscosupplementation. b Fluoroscopic image of the needle insertion in the hip joint space range of motion of the hip (5 points) and absence of muscle contractures and length discrepancy (4 points). All side effects and complications of viscosupplementation were noted. In April 2005, all patients were contacted for followup assessment over the phone VAS and HHS.

\section{Statistical analysis}

Software (MS Excel) ${ }^{\circledR}$ was used to collect the data. Statistical analyses were of two kinds, first the differences in HHS and VAS pre-infiltration and post-infiltration (in the three groups) were compared using the paired $t$ test and with the Wilcoxon paired test in the Synvisc group because this group is too small to analyse with the paired $t$ test.

The second comparison was made between the three groups (Synvisc, Synocrom and Adant). The differences in HHS and VAS evolution between the three groups were checked by the analysis of variance statistics with Tukey extension, which also was used for controlling if the groups were initially homogeneous.

The duration of the viscosupplementation effect of the first infiltration was analysed using the Kaplan-Meier survival curves. The delay for the need of hip surgery was also analysed using a Kaplan-Meier survival curve. The statistical significance was set on $P \leq 0.05$.

\section{Results}

The mean pre-infiltration HHS was comparable for the three groups and varied from 64.8 points in the Adant group to 66.8 points in the Synocrom-group. The post-infiltration HHS increased with 6.3 points in the Adant group $(P<0.001)$, with 10.6 points in the Synocrom group $(P<0.05)$ and with 6.1 points in the Synvisc-group $(P>0.05$; Table 2$)$. There was no statistical significant difference in the evolution of the HHS between the three groups (Table 2).

Viscosupplementation provided a highly significant pain reduction in the Adant-group $(P<0.0001)$, a significant pain reduction in the Synocrom-group $(P<0.05)$ and a pain reduction that did not reach significance in the Synviscgroup $(P>0.05)$. There was no significant difference in pain relief between the three treatment products (Fig. 2, Table 3).

Table 2 Evolution in average HHS score

\begin{tabular}{lllll}
\hline & HHS pre & HHS post & Difference & $P$ \\
\hline Adant & $64.8 \pm 13.8$ & $71.1 \pm 15.7$ & +6.3 & $<0.001$ \\
Synocrom & $66.8 \pm 13.8$ & $77.4 \pm 14.7$ & +10.6 & $<0.05$ \\
Synvisc & $66.3 \pm 13.5$ & $72.4 \pm 14.5$ & +6.1 & $>0.05$ \\
\hline
\end{tabular}



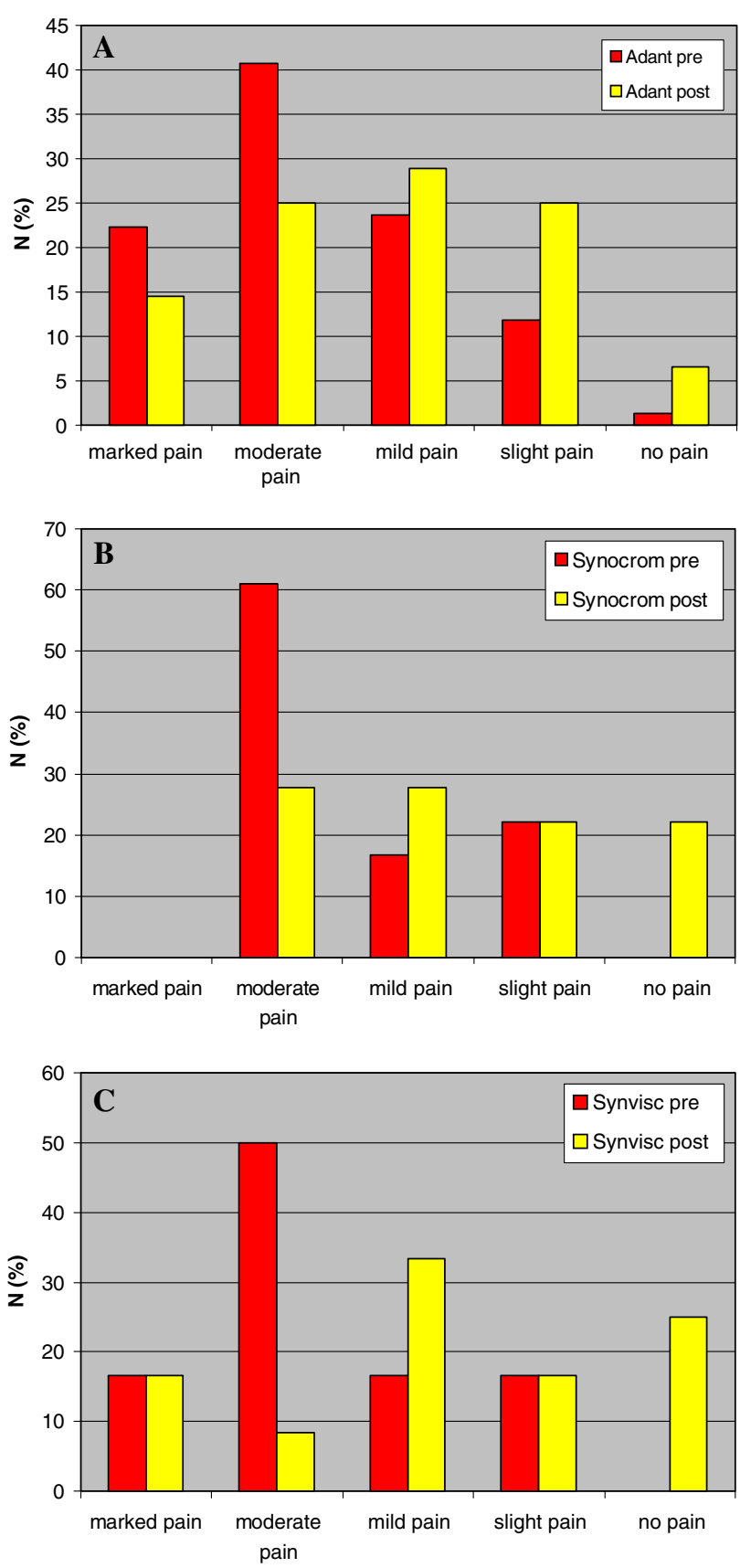

Fig. 2 Pain relief in the three treatment groups a Adant, b Synocrom and $\mathbf{c}$ Synvisc

The duration of the effect of the first infiltration in the three groups is shown in a Kaplan-Meier curve (Fig. 3). The first infiltration was the starting point. Endpoints were the second infiltration or operation of the afflicted hip, or when these were not applicable the latest patient contact, which can be considered as ongoing effect.

There is no significant difference between the three groups $\left(\chi^{2}=0.988\right.$ and $\left.P=0.61\right)$. Figure 4 illustrates the duration of effect of the three groups globally.

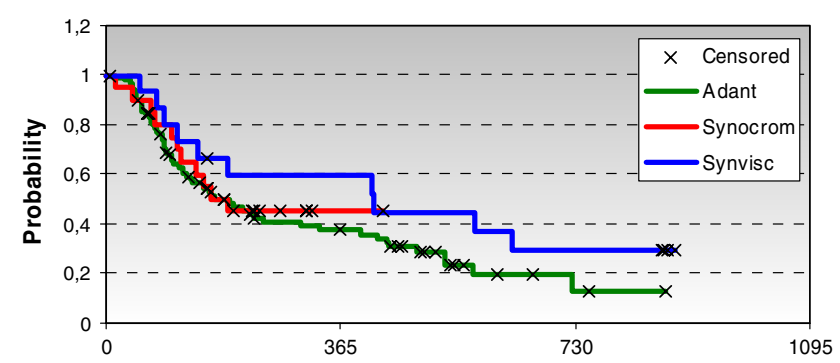

Fig. 3 Kaplan-Meier survival curve of the duration of effect of the first infiltration in days for the three different treatment groups

The positive effect was still ongoing in 46 hips, while in 80 hips patients had either received a second infiltration or THA at, the end of the study (Table 4).

The delay in performing a hip operation is analysed using a Kaplan-Meier survival curve (Fig. 4). After 3 years, $51 \%$ of the patients have not undergone surgery. Because of the comparable outcome achieved with the three HA formulations, we did not differentiate between the three products in this survival analysis (Fig. 5).

\section{Discussion}

The patients attending our outpatient clinic, who were judged eligible for THA, were offered the possibility of a VS therapy. Hundred twenty patients choose this treatment option and 126 hips were treated with Adant, Synocrom or Synvisc. All studies, published up till now, studying the effect of VS in the treatment of hip OA were performed in small patient groups (12-57 patients) [6,9].

The three preparations provided a significant pain relief and improvement of the HHS. The isolated Synvisc group never reached statistical significance in HHS score evolution and VAS during walk test after VS treatment; possibly due to the small number of patients $(N=15)$ in this group. As in the study of Tikiz [13], we found no significant difference between lower and higher molecular weight hyaluronic acids.

We could identify two groups of responders, the ongoing responders who last on average for 352 days after the first infiltration. The second group, where patients either received a second infiltration or THA had an average duration of effect of 174 days.

The VS method is widely used for OA of the knee joint, but there are only a few studies about its use in OA of the hip. Most authors agree that there should be a role for viscosupplementation in the treatment of hip OA. [1-3, 6, 7, 9-11] Our findings confirm the effect of VS in patients suffering OA of the hip. To our knowledge, this is the largest series of patients with hip osteoarthritis treated with viscosupplementation. There are no guidelines regarding 
Fig. 4 Kaplan-Meier survival curve of the duration of effect of the first infiltration in days (three treatment groups together)

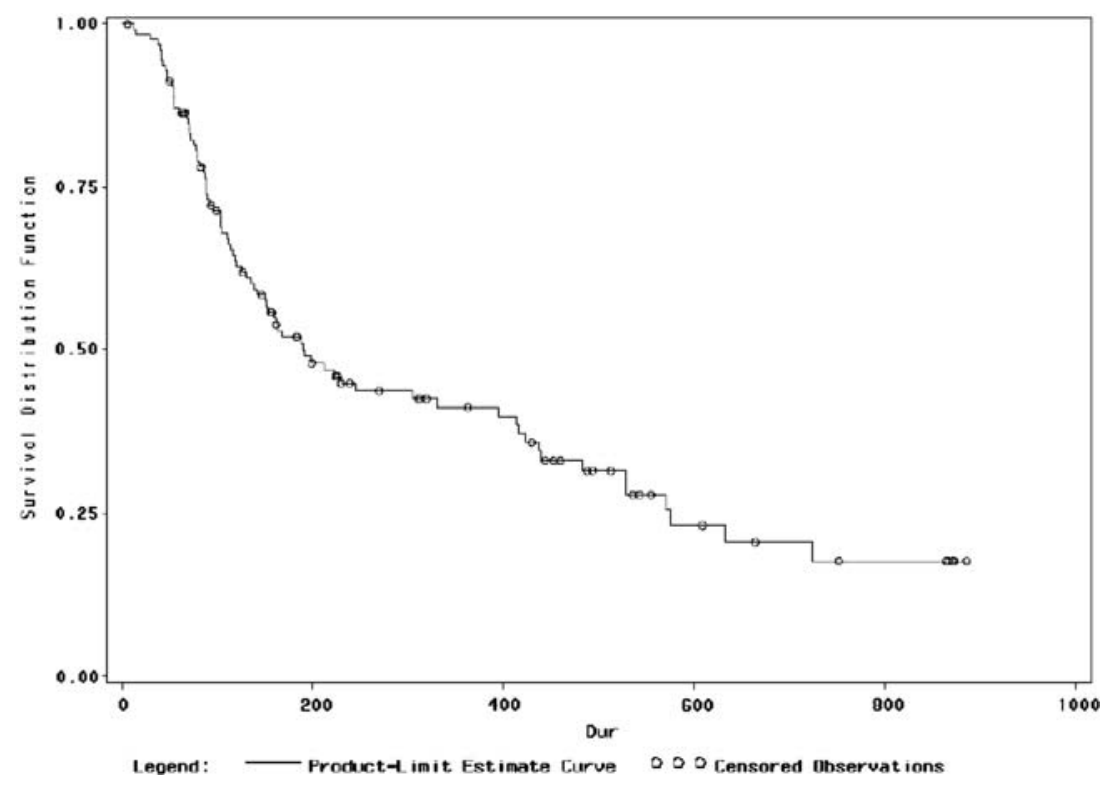

Table 3 Evolution in average VAS pain during walking score

\begin{tabular}{lllll}
\hline & VAS pre & VAS post & Difference & $P$ \\
\hline Adant & $51 \pm 23$ & $39 \pm 27$ & -12 & $<0.0001$ \\
Synocrom & $43 \pm 22$ & $29 \pm 23$ & -14 & $<0.02$ \\
Synvisc & $47 \pm 26$ & $30 \pm 29$ & -17 & $>0.05$ \\
\hline
\end{tabular}

Pre: at first infiltration

Post: at follow-up

the optimal number of injections needed to obtain optimal clinical response, which seems to be different $[1,2,8,10$, 11] between products and dependent on the molecular weight (MW) [7]. We evaluated the time to relapse of one infiltration.

Other authors advocated the use of ultrasound for controlling the needle position in the hip joint. [9, 3] In our series, we performed the infiltrations under fluoroscopy, in experienced hands this approach has proven to be accurate and safe. We use a very low amount of contrast liquid to avoid dilution of the product.

Of all the reports published, we have the longest followup, up to approximately 3 years. The analysis by means of the Kaplan-Meier survival curve learns that there is a $50 \%$ probability of long-term effect (more than 2 years).

We saw no infectious adverse events and no serious systemic reactions, but all the interventions are performed in the operating theatre under strict aseptic conditions. The adverse events rates ranged from 10 to $30 \%$ which is slightly higher than the rates reported in VS treatment of knee OA [7]. Repeated injections did not increase the risk of adverse events [7]. Some patients experienced transient hip pain after the infiltration but made a full recovery in the next days. In the study of Conrozier [6], transient hip pain was reported after $10.1 \%$ of the injections. The products used in this study were all well tolerated. Complications are rare but a single case of septic arthritis was reported after multiple injections of hyaluronate and glucocorticoid [5]. Gout, pseudogout and chondrocalcinosis have not been reported after hip infiltrations.

Intra articular injection of hyaluronic acid can provide long-term pain relief and improvement of joint function even in patients eligible for THA. Despite the use of fluoroscopy, this technique can be performed in an outpatient clinic, allowing patients to return home on the day of the intervention. At present, viscosupplementation therapy for OA of the hip is only palliative. It can be an alternative for young THA candidates, patients with surgical contra-indications and patients in whom NSAID use is not appropriate. There is only 1 randomised, double blind, placebo controlled trial of patients with hip OA [15]. The study was designed with a three-armed parallel-group (Hyalgan vs. methylprednisolone vs. lidocaine). One hundred and one were treated. There was no statistically significant difference between the three products on any outcome measure including the primary outcome measure, i.e., 'Pain on walking', at three 3 months of follow-up.

The results of our study should be considered in the light of the limitations of the design of this study. It is a non placebo controlled non randomised prospective study. It is known from experience with knee OA that the placebo effect of VS tends to be substantial [8]. The dimension of the groups treated with the three different products differs from 15 to 91 patients.

Viscosupplementation with hyaluronic acid seems to be a valuable technique for the management of painful OA of 
Fig. 5 Kaplan-Meier survival curve for the delay to surgery in days for the three groups confounded

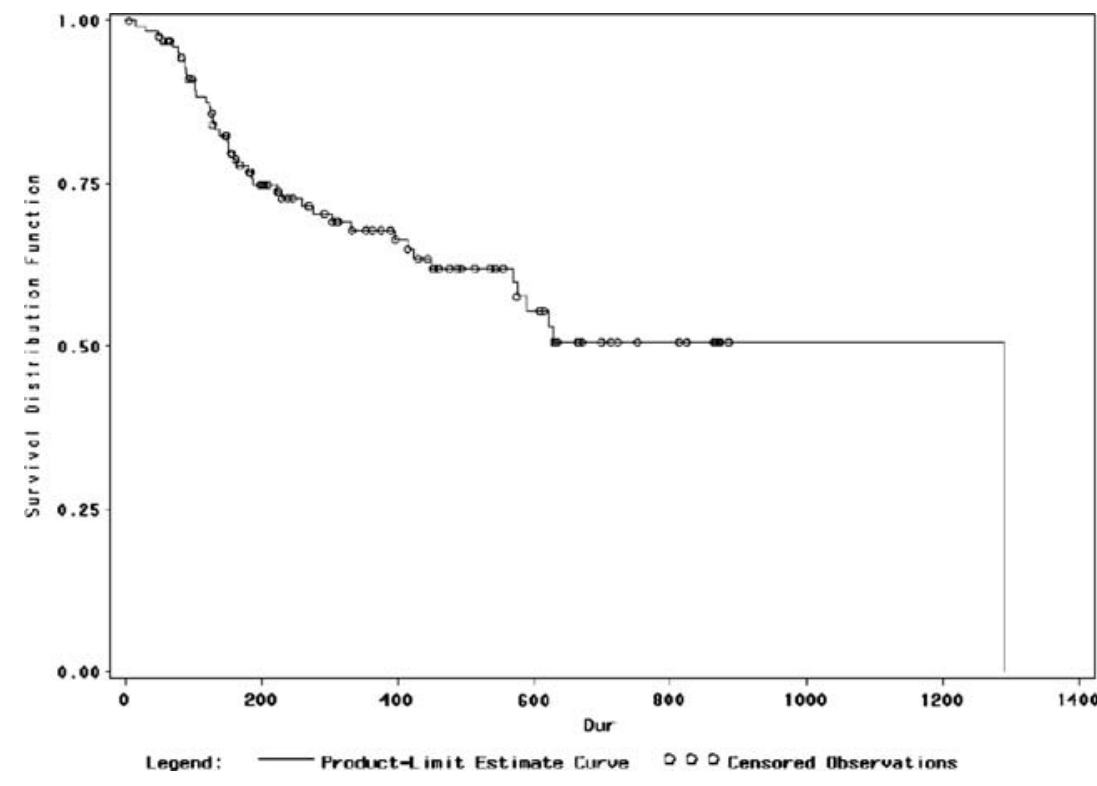

Table 4 Duration of treatment effect after first infiltration

\begin{tabular}{lcc}
\hline & Patients & Average duration (days) \\
\hline $\begin{array}{l}\text { Effect of 1st infiltration } \\
\text { still going on }\end{array}$ & 46 & $352 \pm 258$ \\
(range 6-886) \\
$\begin{array}{l}\text { Effect of 1st infiltration } \\
\text { terminated }\end{array}$ & 80 & $174 \pm 163$ \\
(range 13-724)
\end{tabular}

the hip that may delay the need for surgical intervention. Further prospective randomised placebo controlled studies are necessary to draw definite conclusions.

Ackowledgments The authors are grateful to N. Vandenhecke for her help in the preparation of this manuscript and to J. Nijs for assistance in the statistical analysis of the data. No sources of funding were used to assist in the preparation of this study. The authors have no conflicts of interest that are directly relevant to this article.

\section{References}

1. Berg P, Olsson U (2004) Intra-articular injection of non-animal stabilised hyaluronic acid (NASHA) for osteoarthritis of the hip: a pilot study. Clin Exp Rheumatol 22(3):300-306

2. Bragatini A, Molinaroli F (1984) A pilot clinical evaluation of the treatment of hip osteoarthritis with hyaluronic acid. Clin Ther Res 55:3319-3330

3. Caglar-Yagci H, Unsal S, Yagci I, Dulgeroglu D, Ozel S (2005) Safety and efficacy of ultrasound-guided intra-articular hylan G-F 20 injection in osteoarthritis of the hip: a pilot study. Rheumatol Int 25(5):341-344

4. Callaghan JJ, Albright JC, Goetz DD, Olejniczak JP, Johnston RC (2000) Charnley total hip arthroplasty with cement. Minimum twenty-five-year follow-up. J Bone Joint Surg Am 82(4):487-497

5. Chazerain P, Rolland D, Cordonnier C, Ziza JM (1999) Septic hip arthritis after multiple injections into the joint of hyaluronate and glucocorticoid. Rev Rhum Engl Ed 66(7-9):436
6. Conrozier T, Bertin P, Mathieu P, Charlot J, Bailleul F, Treves R, Vignon E, Chevalier X (2003) Intra-articular injections of hylan G-F 20 in patients with symptomatic hip osteoarthritis: an openlabel, multicentre, pilot study. Clin Exp Rheumatol 21(5):605-610

7. Conrozier T, Vignon E (2005) Is there evidence to support the inclusion of viscosupplementation in the treatment paradigm for patients with hip osteoarthritis? Clin Exp Rheumatol 23(5):711716

8. Kirwan J (2001) Is there a place for intra-articular hyaluronate in osteoarthritis of the knee? Knee 8(2):93-101

9. Migliore A, Martin LS, Alimonti A, Valente C, Tormenta S (2003) Efficacy and safety of viscosupplementation by ultrasound-guided intra-articular injection in osteoarthritis of the hip. Osteoarthritis Cartilage 11(4):305-306

10. Migliore A, Tormenta S, Martin LS, Valente C, Massafra U, Granata M, Alimonti A (2005) Open pilot study of ultrasound-guided intra-articular injection of hylan G-F 20 (Synvisc) in the treatment of symptomatic hip osteoarthritis. Clin Rheumatol 24(3):285-289

11. Migliore A, Tormenta S, Valente C, Massafra U, Martin Martin LS, Carmenini E, Bernardini A, Alimonti A (2005) Intra-articular treatment with Hylan G-F 20 under ultrasound guidance in hip osteoarthritis. Clinical results after 12 months follow-up. Reumatismo 57(1):36-43

12. Rossignol M, Leclerc A, Allaert FA, Rozenberg S, Valat JP, Avouac B, Coste P, Litvak E, Hilliquin P (2005) Primary osteoarthritis of hip, knee, and hand in relation to occupational exposure Occup Environ Med 62(11):772-777

13. Tikiz C, Unlu Z, Sener A, Efe M, Tuzun C (2005) Comparison of the efficacy of lower and higher molecular weight viscosupplementation in the treatment of hip osteoarthritis. Clin Rheumatol 24(3):244-250

14. Bellamy N, Campbell J, Robinson V, Gee T, Bourne R, Wells G (2005) Viscosupplementation for the treatment of osteoarthritis of the knee. Cochrane Database Syst Rev Issue 2. doi: 10.1002/ 14651858.CD005321

15. Qvistgaard E, Christensen R, Torp-Pedersen S, et al (2006) Intraarticular treatment of hip osteoarthritis: a randomised trial of hyaluronic acid, corticosteroid, and isotonic saline. Osteoarthritis Cartilage 14(2): 163-170 